\title{
A Multiple Sclerosis Patient Benefitting from CSF Flow Enhancement Had Imaging Signs of "Glymphatic-Lymphatic System Disease." Case Report
}

\author{
Antonio Scollato \\ Cardinale Panico Hospital, Tricase \\ Francesco Lolli \\ University of Florence, Italy \\ Giancarlo Lastrucci \\ Cardinale Panico Hospital, Tricase

\section{Anna Repice}

Careggi University Hospital, Florence

\section{Giuseppe De Santis}

Cardinale Panico Hospital, Tricase

\section{Claudio Nicoletti}

University of Florence, Florence

\section{Berardino Porfirio}

University of Florence, Italy

Pasquale Gallina ( $\nabla$ pasquale.gallina@unifi.it)

University of Florence

\section{Case report}

Keywords: CSF dynamics disturbances, CSF shunting, glymphatic system, multiple sclerosis

Posted Date: November 22nd, 2021

DOI: https://doi.org/10.21203/rs.3.rs-1067351/v1

License: @ (i) This work is licensed under a Creative Commons Attribution 4.0 International License. Read Full License 


\section{Abstract}

\section{Background}

The derangement of CSF circulation impacts the functions of glymphatic-lymphatic system (G-Ls), which regulates solute trafficking and immune surveillance in the CNS. The imaging features of dilated perivascular spaces imply the substantial impairment of the G-Ls and can be easily estimated It has been proposed that multiple sclerosis can be viewed as a disease involving a failure component and therapeutically could be targeted as such.

Case presentation

We report the case of a female patient diagnosed with primary progressive multiple sclerosis, also presenting perivascular spaces dilatation, who transiently improved after CSF shunt diversions.

\section{Conclusions}

The G-Ls failure leads to dysregulation of waste molecule clearance in the brain and an altered CNS immune response, potentially in many diseases. The clinical improvement observed in our patient may relate to an increased clearance of inflammatory mediators following the G-Ls reestablishment obtained by CSF flow enhancement.

\section{Background}

The glymphatic-lymphatic system (G-Ls) regulates solute trafficking and immune surveillance in the CNS, sub-serving the flow of CSF from the subarachnoid spaces into the perivascular areas and subsequently into the interstitium, with the aquaporin- 4 water channels running it. ${ }^{1,2}$ The cerebrospinal-interstitial fluid then passes to the venous perivascular and perineural spaces, lastly draining toxic molecules and immune cells from the brain into meninges and deep lymph nodes. Derangements in intra-extracranial hydrodynamics at various levels lead to failure of the CSF dynamic, resulting in G-Ls failure, ${ }^{1}$ which possibly configures "G-Ls pathology."3 The characteristic imaging pattern of G-Ls dysfunction is perivascular space dilatation caused by internal congestion and stagnation with an accumulation of cerebrospinal-interstitial fluid. ${ }^{4}$

It is emerging that neuroinflammatory diseases are associated with G-Ls dysfunction. ${ }^{5}$ Dilated perivascular spaces occur more frequently in multiple sclerosis (MS) than in healthy controls. ${ }^{6,7}$ In an experimental model of autoimmune encephalitis, perivascular aquaporin-4 localization is lost. ${ }^{8}$ Inflammation disrupts the association of astrocytes with blood vessels and surrounding neurons in MS. ${ }^{9}$ Inflammatory follicle-like aggregates in progressive MS are located in these perivascular spaces. ${ }^{10}$ The complex of these findings suggests that improving G-Ls flow in an MS patient with perivascular space dilatation, forcing CSF circulation through the diversion as described in other models, ${ }^{11}$ could favor the clearance of inflammatory mediators in MS, ${ }^{12}$ and thus potentially improving the clinical picture. 


\section{Case Presentation}

A 34-year-old female developed headaches, gait disturbances, and urinary dysfunction over time. In fluidattenuated inversion recovery images of the brain and spinal cord, magnetic resonance imaging (MRI) revealed diffuse hyperintense T2 nodular lesions affecting the paraventricular and subcortical white matter. At least two of these lesions showed gadolinium enhancement in T1-weighted images. The blood tests were within physiological parameters, while the neurophysiological examination revealed changes in visual evoked potential and somatosensory evoked potential from the left median nerve. Oligoclonal IgG bands at IEF occurred in the CSF. The clinical and paraclinical findings led to the diagnosis of MS. Methylprednisolone offered her some improvement. Immunomodulatory treatments with interferon-beta and glatiramer acetate were started and later discontinued due to intolerance. After 16 months of continuous neurological impairment, the patient was diagnosed with primary progressive MS. An MRI performed during the same period revealed a stable picture. She was voluntarily tested in another hospital for cerebrospinal venous insufficiency in 2011. A severe cerebrospinal venous insufficiency was diagnosed, and she underwent percutaneous transluminal angioplasty at the level of the internal jugular veins. She had about two years of clinical benefit after the procedure, which paralleled normal anatomic and functional conditions of jugular veins according to echocolor Doppler examination and stability of MS lesions at MRI. Subsequently, her neurological condition deteriorated gradually. Sonographic findings suggested a recurrence of chronic cerebrospinal venous insufficiency after two years. At 38, the patient came under our observation with a disease duration of 4 years (video 1). Her Kurtzke Expanded Disability Status Scale (EDSS) was 6.5. MRI showed typical MS lesions as well as enlarged perivascular spaces (Fig. 1), as classified according to Wuerfel et al. ${ }^{7}$ Considering the severe progression of the disease, after a full appraisal by the Ethical Committee, the patient gave written consent to undergo a program of CSF diversions as compassionate treatment. She received two one-day external lumbar drainages (12-15 $\mathrm{mL} / \mathrm{h}$ over 24 hours). ${ }^{13}$ Immediately after the drainages, the patient experienced marked clinical improvement (video 2) and EDSS 3.0 a week after both procedures. This status lasted about two months after the first drainage and about one month after the second one. Her headaches, in particular, vanished, and she regained her gait and urinary functions. MRI detected a dimensional increase of the subcortical right frontal MS lesion after xx months. The neurological benefit lasted approximately two months after the first drainage and about one month after the second. In September 2014, after the recurrence of headache, urinary and gait disturbances and a mild cognitive impairment (Mini-Mental State Examination score of 25), the EDSS score reached 8.0, and we implanted a lumboperitoneal shunt (Spetzler system, Integra LifeSciences Corp). After the intervention, the patient improved in a few hours. She had mild spastic-ataxic paraparesis with full autonomy in gait, sphincter recovery, and headache resolution. In the same period, MRI revealed the disappearance of contrast enlargement of the brain lesions, while lesion enlargement appeared at the T6-T7 level. Further improvement was progressively observed, but a slow worsening occurred five months after surgery. A year after, an MRI revealed the absence of active MS lesions and the persistence of perivascular space enlargement. Thirteen months after surgery, she received an EDSS score of 7.5. An ophthalmological examination revealed severe right optic impairment. The patient refused further external lumbar drainage to verify the hypothesis that clinical worsening was 
due to shunt failure. In October 2018, her MRI was stable, with no signs of activity, and her neurological conditions remained unchanged.

\section{Discussion And Conclusions}

Being aware of the anecdotal nature, potential placebo effect, and possibly unrelated and unknown factors, this case suggests that CSF diversions may have played a role in this MS patient's transient clinical improvement. CSF diversion may have bolstered interstitial fluid dynamics by decreasing interstitial brain pressure and decongesting perivascular spaces. Our procedure assumed that an impaired CSF flow altered the G-Ls control in removing proinflammatory molecules from CSF and local inflammation in a congested perivascular space. The observation that perivascular spaces remained enlarged upon imaging after CSF diversion is not surprising because post-inflammatory fibrosis might make perivascular enlargement a permanent feature. ${ }^{14}$ Furthermore, as previously hypothesized by Louveau et al., ${ }^{5}$ modulation of CSF drainage through the meningeal lymphatic vasculature will reduce the quantity of CNS antigens entering the related lymph nodes, resulting in down-regulation of the autoimmune response. An overall combination of "hydraulic" and anti-inflammatory effects must be ruled in this scenario. The timing of neurological recovery, on the other hand, seems to support the "hydraulic" mechanism, as the advantage appeared quickly after CSF drainage and vanished in a few days, as in typical normal pressure hydrocephalus. ${ }^{15}$ Notably, the dominant signs in our patient before diversions (i.e. gait and urinary disturbances, slight cognitive deterioration, and headache) are also characteristic of a normal pressure hydrocephalus situation and are sensitive to CSF shunting. ${ }^{16}$ Lumbar-peritoneal shunting induced a beneficial effect that lasted only for a limited time. One possibility is that following insufficiency of CSF flux drainage reduced/suppressed the treatment's efficacy.

By no means are we advocating for a new MS therapy. However, this report represents an exploratory approach to a disease whose pathogenesis is still unknown and for which there is no definitive cure. Furthermore, given what we now know about G-Ls dysfunction as associated to CSF flow disturbances, ${ }^{1}$ this knowledge may incentivize research into restoring CSF dynamics in all conditions where toxic molecules and immune cell clearance are inadequate.

\section{Declarations}

Ethics approval and consent to participate

The patient gave written consent to undergo all the medical procedures she received after appraisal by Alessandro Manzoni, Lecco, Italy; Ethical Committee.

Consent for publication

The patient gave consent for publication of person's data.

Availability of data and materials 
The clinical data are not publicly available due to patient's privacy protection but are available from the corresponding author on reasonable request.

Competing interests

The authors declare that they have no competing interests.

Funding

This paper did not receive funding.

Authors' contributions

$A S, B P$ and $P G$ have made substantial contribution to the conception of the study, $P G, F L, B P, A R, G L, C N$ and GDS have drafted the work or substantially revised it.

All authors have approved the submitted version have agreed both to be personally accountable for the author's own contributions and to ensure that questions related to the accuracy or integrity of any part of the work, even ones in which the author was not personally involved, are appropriately investigated, resolved, and the resolution documented in the literature.

\section{Acknowledgement}

The authors thank Dr Andrea Rosi, neuroradiologist (Geneva University Hospital, Geneva, Switzerland), for assistance in analyzing imaging data.

\section{References}

1. Nedergaard M, Goldman SA. Glymphatic failure as a final common pathway to dementia. Science 2020;370(6512): 50-56.

2. Louveau A, Herz J, Alme MN, Salvador AF, Dong MQ, Viar KE et al. CNS lymphatic drainage and neuroinflammation are regulated by meningeal lymphatic vasculature. Nat. Neurosci. 2018;21(10):1380-1391.

3. Gallina P, Nicoletti C, Scollato A, Lolli F. The "Glymphatic-Lymphatic System Pathology" and a New Categorization of Neurodegenerative Disorders. Front. Neurosci. 2021;15:669681.

4. Wardlaw J, Nemveniste H, Nedergaard M, Zlokovic BV, Mestre H, Lee H et al. Perivascular spaces in the brain: anatomy, physiology and pathology. Nat. Rev. Neurol. 2020;16(3):137-153.

5. Louveau A, Da Mesquita S, Kipnis J. Lymphatics in neurological disorders: a neuro-lympho-vascular component of multiple sclerosis and Alzheimer's disease? Neuron 2016;91(5):957-973.

6. Etemadifar M, Hekmatnia A, Tayari N, Kazemi M, Ghazavi A, Akbari M, et al. Features of VirchowRobin spaces in newly diagnosed multiple sclerosis patients. Eur. J. Radiol. 2011;80(2):e104-108. 
7. Wuerfel J, Haertle M, Waiczies H, Tysiak E, Bechmann I, Wernecke KD et al. Perivascular spaces-MRI marker of inflammatory activity in the brain? Brain 2008;131(Pt9):2332-2340.

8. Wolburg-Buchholz K, Mack AF, Steiner E, Pfeiffer F, Engelhardt B, Wolburg H. Loss of astrocyte polarity marks blood-brain barrier impairment during experimental autoimmune encephalomyelitis. Acta Neuropathol. 2009;118(2):219-233.

9. Eilam R, Segal M, Malach R, Sela M, Arnon R, Aharoni R. Astrocyte disruption of neurovascular communication is linked to cortical damage in an animal model of multiple sclerosis. Glia 2018;66(5):1098-1117.

10. Lassmann H: Multiple sclerosis pathology. Review. Cold Spring Harb Perspect Med 2018;8: pii: a028936.

11. Abolfazli E, Fatouraee N, Seddighi AS. Effects of lumbar drainage on CSF dynamics in subarachnoid hemorrhage condition: A computational study. Comput. Biol. Med. 2016;77:49-58.

12. Khaibullin T, Ivanova V, Martynova E, Cherepnev G, Khabirov F, Granatov E. Elevated levels of proinflammatory cytokines in cerebrospinal fluid of multiple sclerosis patients. Front. Immunol. 2017,8:531.

13. Gallina P, Lastrucci G, Caini S, Di Lorenzo N, Porfirio B, Scollato A. Accuracy and safety of 1-day external lumbar drainage of CSF for shunt selection in patients with idiopathic normal pressure hydrocephalus. J. Neurosurg. 2018;1-7.

14. Inglese M, Bomsztyk E, Gonen O, Mannon LJ, Grossman RI, Rusinek H. Dilated perivascular spaces: hallmarks of mild traumatic brain injury. AJNR Am. J. Neuroradiol. 2005;26(4):719-724.

15. Lenfeldt N, Hansson W, Larsson A, Birgander R, Eklund A, Malm J. Three-day CSF drainage barely reduces ventricular size in normal pressure hydrocephalus. Neurology 2012;79(3):237-242.

16. Adams RD, Fisher CM, Hakim S, Ojemann RG, Sweet WH. Symptomatic occult hydrocephalus with "normal" cerebrospinal-fluid pressure. A treatable syndrome. N. Engl. J. Med. 1965;273:117-126.

\section{Figures}




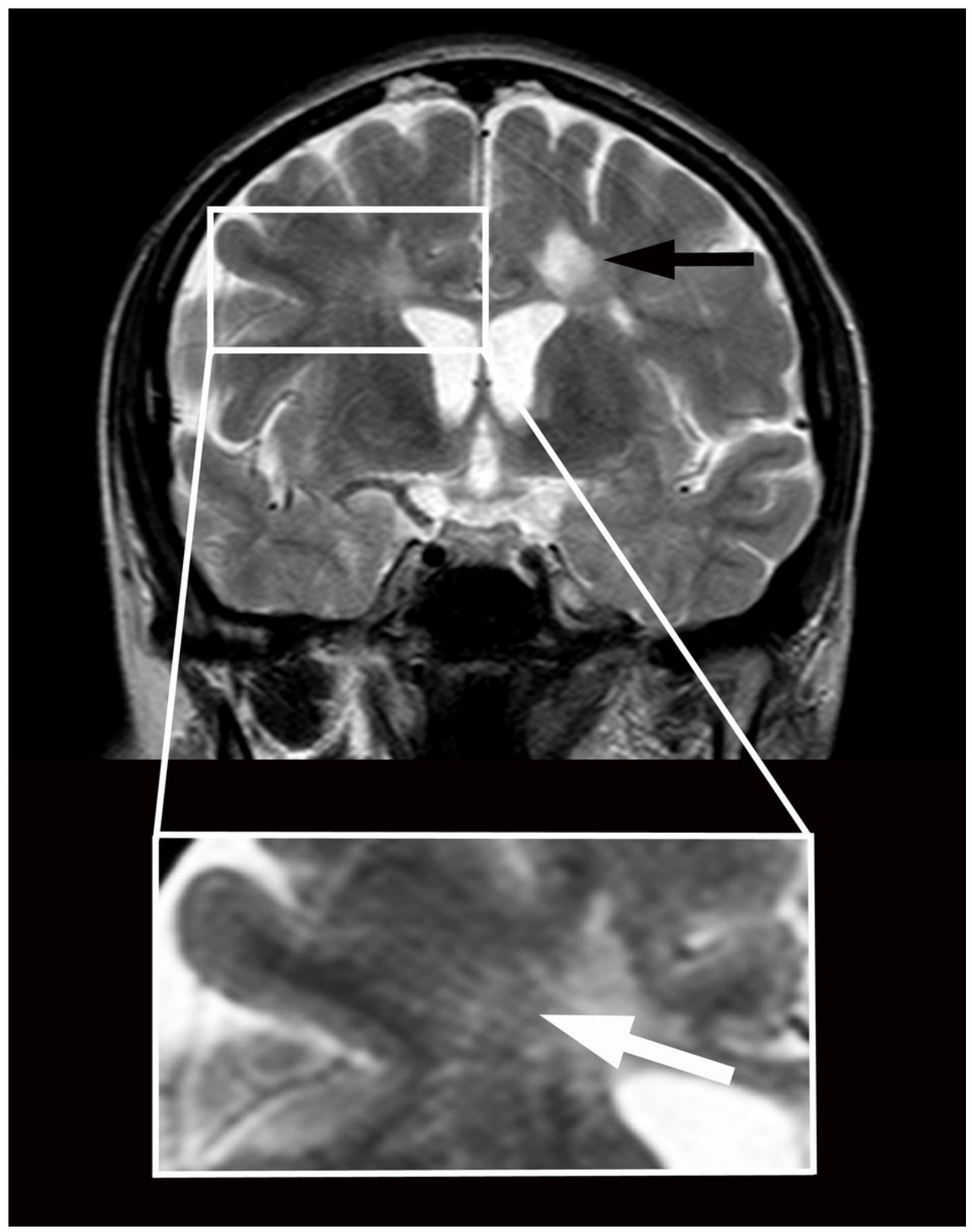

\section{Figure 1}

Magnetic resonance imaging in a patient with primary progressive multiple sclerosis and features of brain interstitial space congestion. Imaging was performed before positioning a lumbar-peritoneal shunt. Multiple sclerosis lesions appear as round hyperintensities on coronary T2-weighted imaging obtained at the level of the third ventricle (black arrow). Enlarged perivascular spaces appear as linear 
hyperintensities crossing radially the Corona Radiata from the cortex to the ventricles (white arrow in higher magnification below). The size of the ventricles is normal.

\section{Supplementary Files}

This is a list of supplementary files associated with this preprint. Click to download.

- Video1M.mpg

- Video2M.mpg

- Video3M.mpg 